\title{
IPHE Regulations Codes and Standards Working Group - Type IV COPV Round Robin Testing
}

\author{
Maes, M., Starritt, L.* \\ Zheng, J.Y., Ou, K. ** \\ Keller, J. *** \\ *NASA White Sands Test Facility, United States \\ ** Zhejiang University, China \\ *** Zero Carbon Energy Solutions, Inc., \\ Consultant U.S. DOE, Fuel Cell Technology Office, \\ Safety Codes and Standards Program \\ Previously with Sandia National Laboratories
}

Product of IPHE RCSWG

Brazil, China, European Commission, France, Italy, Germany, Japan, New Zealand, Norway, Russia, South Africa, United Kingdom, United States.

\begin{abstract}
This manuscript presents the results of a multi-lateral international activity intended to understand how to execute a cycle stress test as specified in a chosen standard (GTR, SAE, ISO, EIHP ...). The purpose of this work was to establish a harmonized test method protocol to ensure that the same results would be achieved regardless of the testing facility. It was found that accurate temperature measurement of the working fluid is necessary to ensure the test conditions remain within the tolerances specified. Continuous operation is possible with adequate cooling of the working fluid but this becomes more demanding if the cycle frequency increases. Recommendations for future test system design and operation are presented
\end{abstract}

Corresponding Author:

Dr. Jay Keller, e-mail: Jay.Keller@ZCES-Inc.com

(C) 2016. This manuscript version is made available under the Elsevier user license

http://www.elsevier.com/open-access/userlicense/1.0/ 


\section{Nomenclature}

\begin{tabular}{|l|l|}
\hline Acronym & Definition \\
\hline BPR & Backpressure Regulators \\
\hline${ }^{\circ}$ C & Degrees Centigrade \\
\hline COPV & Composite Overwrapped Pressure Vessel \\
\hline EIHP & European Integrated Hydrogen Project \\
\hline GTR & Global Technical Regulation \\
\hline HDPE & High-Density Polyethylene \\
\hline HVAC & Heating Ventilation and Air Conditioning \\
\hline IPHE & International Partnership for Hydrogen and Fuel Cells in the Economy \\
\hline L & Liter \\
\hline Min & Minutes \\
\hline MPa & Mega Pascal (1.0 x 10 ${ }^{6}$ Pascal) \\
\hline NASA & National Aeronautics and Space Administration \\
\hline NWP & Normal Working Pressure which is the same as Normal Service Pressure \\
\hline RCSWG & Regulations Codes and Standards Working Group of the IPHE \\
\hline RR & Round Robin \\
\hline s & Seconds \\
\hline SAE & Society of Automotive Engineers \\
\hline TMMP & Test Measurement Method Protocol \\
\hline WSTF & White Sands Test Facility \\
\hline
\end{tabular}




\section{Test Measurement Protocol Requirements}

B a ckground

Typically, the purpose of any pressure cycle test is to test the tank for cyclic fatigue, under expected service conditions. The three currently proposed hydraulic pressure test cycle documents, (the EIHP rev12b, SAE J2579 rev 2 draft, and the Global Technical Regulation (GTR)) are very similar with one significant exception relevant to type IV tanks. The EIHP specifies the lower pressure limit to be $\leq 2 \mathrm{MPa}$. The GTR and SAE J2579 rev 2 draft specify the lower limit to be $(2 \pm 1)$ MPa which, is consistent with the automobile controlled lower limit of $2 \mathrm{MPa}$. In addition, no ramp rate for pressurization or depressurization is supplied by any of the proposed tests in the standards or the GTR, with the exception that the overall cycle frequency cannot exceed 10 cycles/min.

\section{Purpose}

The suggested test measurement protocol developed by the RCSWG was established to provide a test measurement method protocol (TMMP) for use in this Round Robin (RR). An outcome of this RR is a test method measurement protocol that when applied around the world, consistency in the test measurements could be assured. It is the goal of the RCSWG that test measurement methodologies developed and proven as a result from performing this internationally harmonized protocol would be applied to the relevant test requirements in the international standards and regulations such as the European Integrated Hydrogen Project (EIHP) CGH2R rev 12b (EIHP rev 12b), SAE J2579 rev 2 draft, and the Global Technical Regulation (GTR) for Hydrogen Fueled Vehicles (ECE-TRANS-WP29-GRSP-2012-12e). It is important to note that a qualifying test sequence is not specified, nor was the goal or objective for the round robin campaign because it is out of scope for the RCSWG to:

- Execute a qualifying test sequence,

- Qualify laboratories to execute test sequences,

- Or design test sequences.

With these ideas in mind, requirements were drafted and distributed to the RCSWG and the two test locations for review and concurrence.

\section{Measurement Protocol Requirements}

The proposed TMMP for hydraulic cycling test as agreed by the RCSWG and test locations required the following, in part or whole:

- The static pressure in the tank is to cycle between a specified minimum and maximum value until failure or a specific number of cycles have been executed whichever occurs first.

- The temperature of the fluid in the tank is to be monitored and controlled.

- The temperature of the outside surface of the tank (the skin) is to be monitored and controlled

- The temperature of the ambient is to be monitored and controlled.

- The cycle rate must not exceed $10 / \mathrm{min}$

- The working fluid must be a non-corrosive liquid

Pressure cycle test systems can be constructed either open ended or closed-ended loop for the working fluid. Figure 1 shows configurations for a closed-ended test, Figure 2 shows configurations for open ended test. In this work the tanks tested had end-bosses on both ends, so the dead-ended tank configuration was achieved by putting a plug in one of the end-bosses 


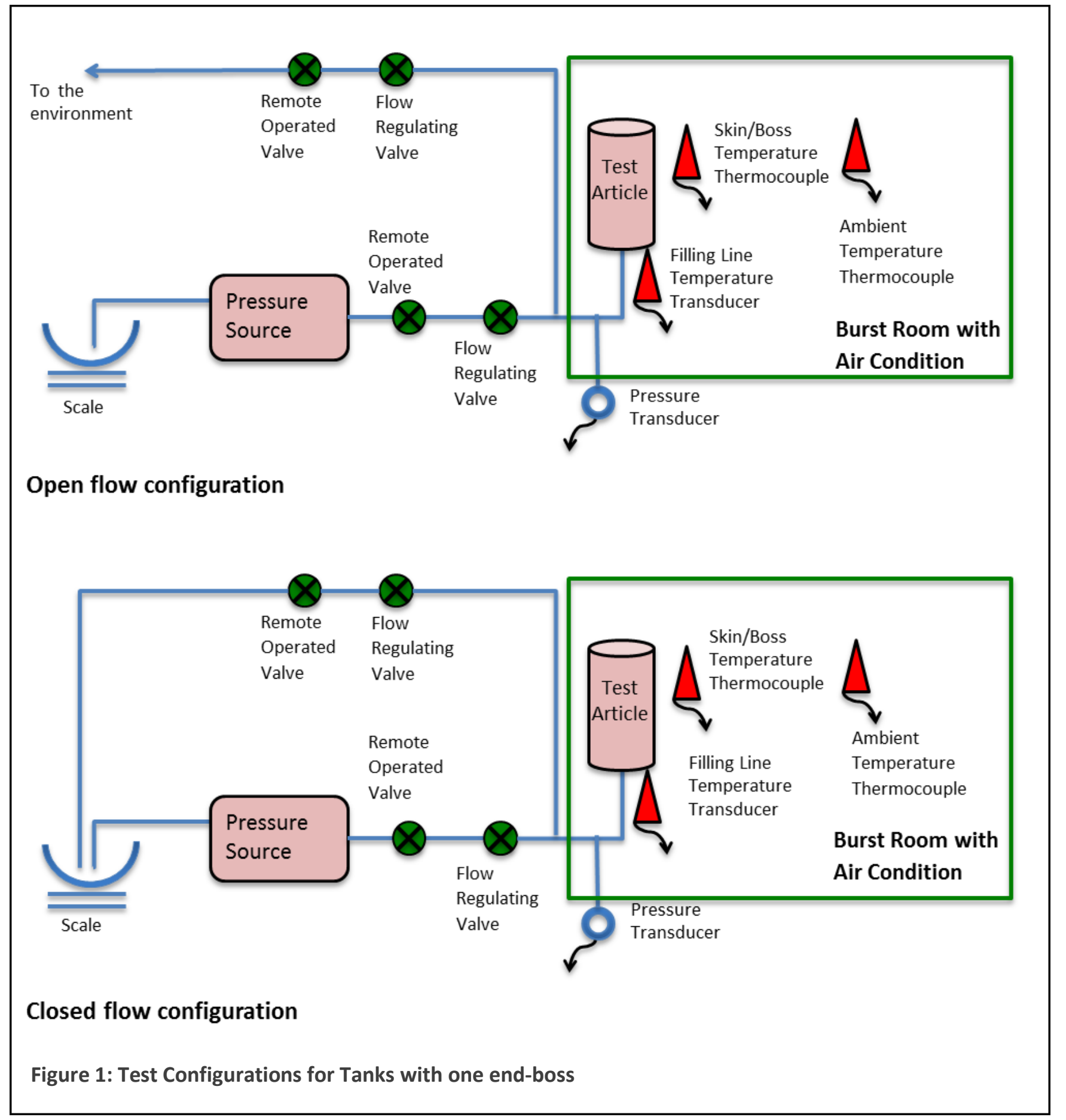




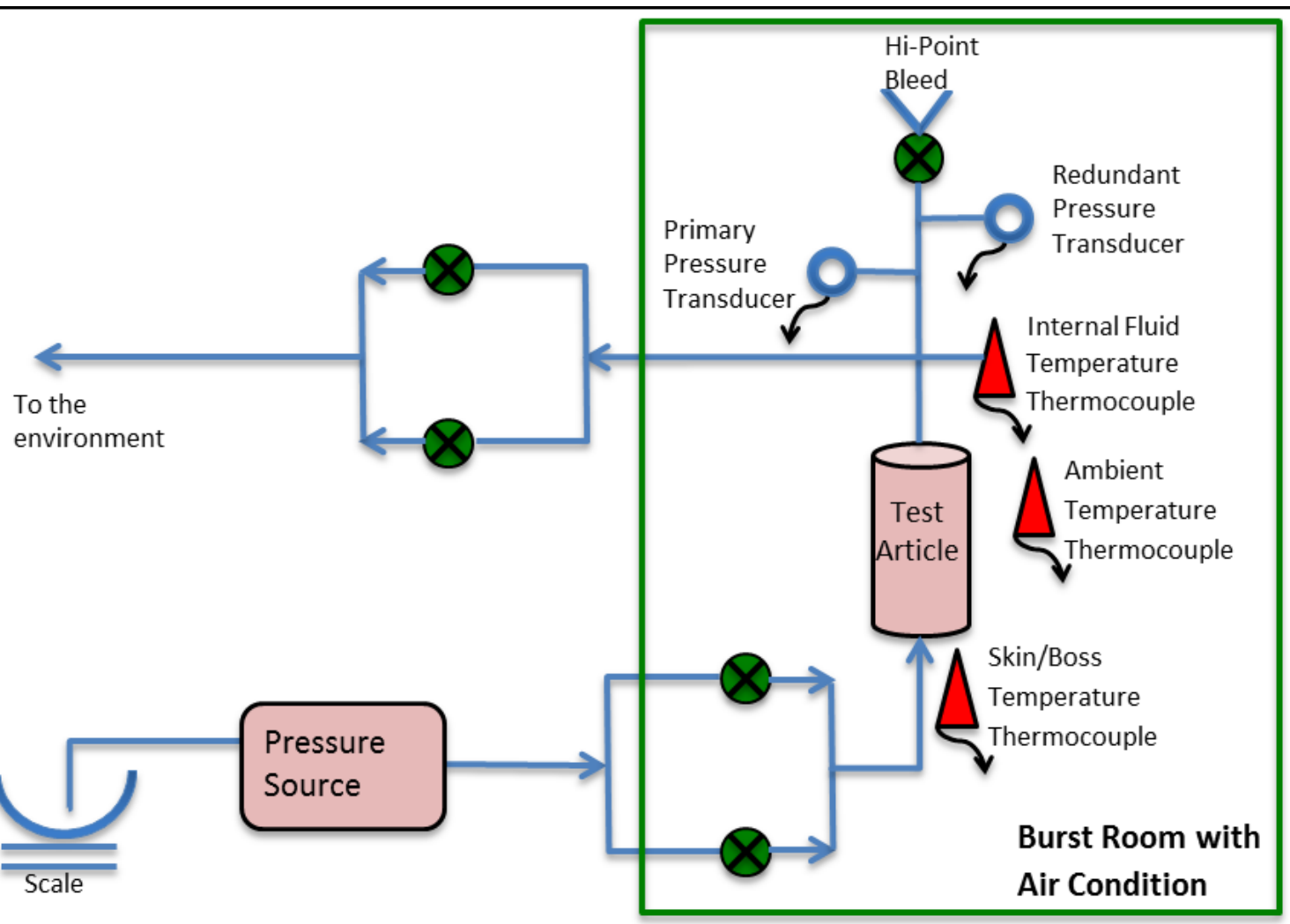

\section{Open flow configuration}

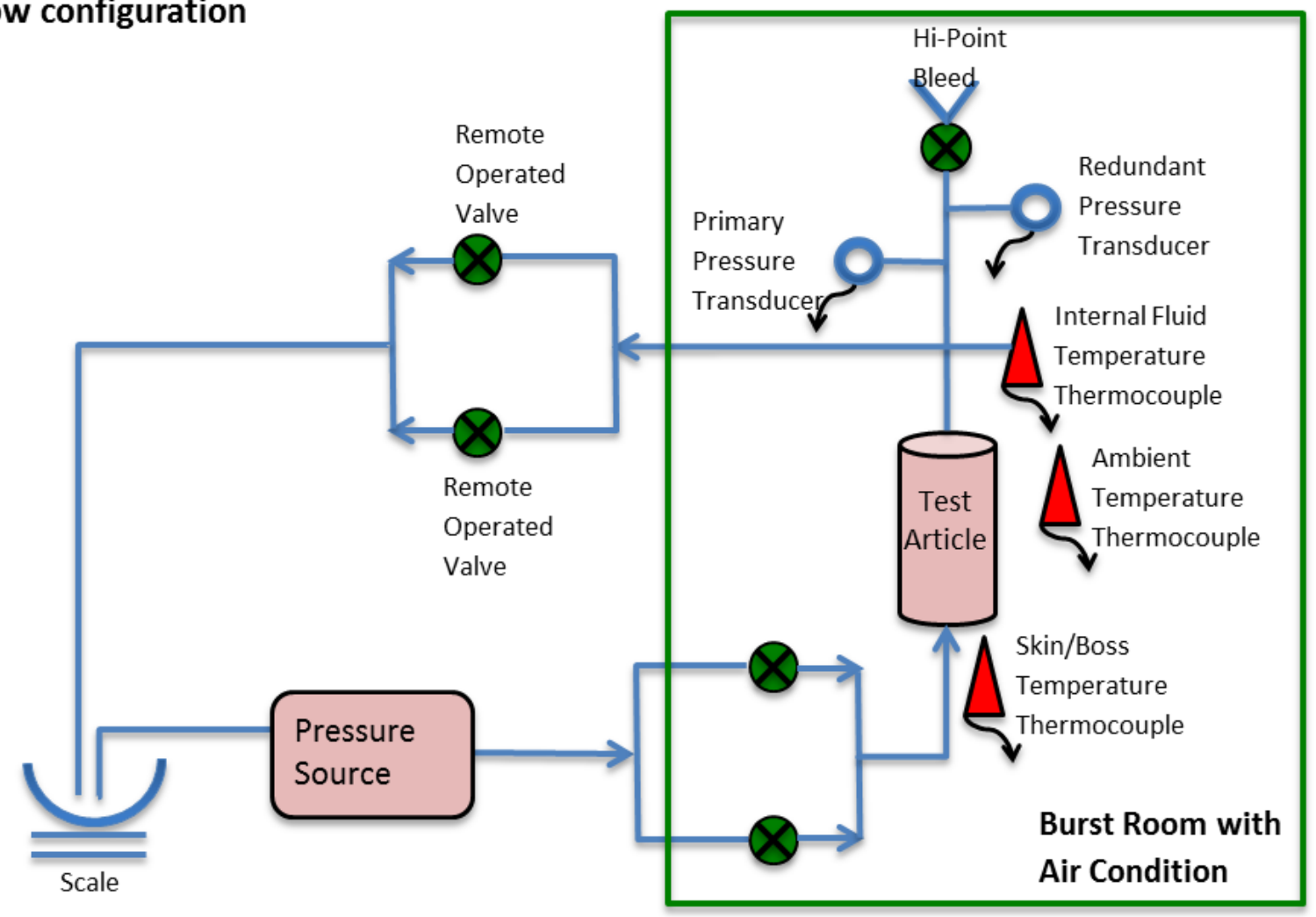

\section{Closed flow configuration}

Figure 2: Test configurations for tanks with two end-bosses 
Below are the requirements on the parameters that were to be measured and controlled during the hydraulic cycling test. These were the recommended practices that were suggested by the RCSWG.

- The static pressure in the tank is measured so no dynamic effects will influence the measurement during both pressurization and depressurization.

- The working fluid temperature in the tank should be measured in several places to understand any temperature variations that might occur - if this is not possible then the temperature of the metal boss at both ends should be made to approximate the internal fluid temperature.

- The temperature of the ambient air is to be measured and controlled

- The temperature of the skin is to be measured

- The data acquisition should be at a rate no slower than $10 \mathrm{~Hz}$.

- The pressure measurement accuracy should be no less than $0.1 \%$ full scale (FS)

- Temperature accuracy $\pm 1^{\circ} \mathrm{C}$

Following some iteration among the RCSWG and with input collected from the Chinese and the U.S. test locations, the suggested TMMP was improved and final requirements given to each test location are documented below. As testing continued some of these requirements were relaxed because of experimental difficulty or it was recognized that it was not necessary for the purpose of this program. The variation of these requirements will be discussed later.

- Pressure range, low: $1 \leq \mathrm{P} \leq 3 \mathrm{MPa}$, high: $125 \% \mathrm{NWP} \leq \mathrm{P} \leq 125 \% \mathrm{NWP}+1 \mathrm{MPa}$

- Dwell time $\geq 0 \mathrm{~s}$ at the minimum pressure

- Dwell time $\geq 1.5 \mathrm{~s}$ at the maximum pressure

- Ambient temperature is to be $(20 \pm 5)^{\circ} \mathrm{C}$

- Cycle frequency is to be $\leq 6 / \mathrm{min}$

- The temperature of the hydraulic fluid within the container is monitored and maintained at the specified temperature: $(20 \pm 5)^{\circ} \mathrm{C}$

- Measurements to $\pm 1^{\circ} \mathrm{C}$ on:

- $\quad$ The metal end boss, both ends

- The skin

- $\quad$ Fluid inside the tank.

- The working fluid temperature can be controlled by modifying the cycle frequency. Reduction of cycle frequency will allow more time for dissipation of heat buildup in the container. Also, a suitable heat exchanger or other temperature control means can be used to maintain the temperature of the fluid in the input lines just before the tank.

- Number of cycles 15,000 (3 X 5000) or failure whichever comes first

- At the end of the cycle sequence, the pressure in the tank is to increase until burst occurs. The static pressure at burst is to be recorded.

For this TMMP, special emphasis and direction was provided to each laboratory to ensure that a minimum pressure was maintained in the event of a premature shutdown. The protocol required that pressures below the ambient pressure (vacuum) in the tank must be avoided at all times (during cycling and idle times) to prevent a liner collapse. In the event that atest needed to be paused or terminated, the tank was to be maintained at the minimum pressure specified by the test until the fluid temperature inside the tank equilibrated to the test requirement of $(20 \pm 5)^{\circ} \mathrm{C}$. Only after thermal equilibrium had been achieved would it be acceptable that the tank pressure be decreased below the minimum test pressure $(2 \pm 1 \mathrm{MPa})$.

To ensure the integrity (cleanliness) of the working fluid and container, the working fluid in the container should be maintained above the ambient pressure $(0.2 \leq \mathrm{P} \leq 0.5 \mathrm{MPa}$ for example). If neither of these is possible then the container needs to be maintained at the ambient pressure. This can be accomplished by having the tank open to 
the ambient pressure conditions, for example; have the "high point bleed" open to the ambient during the idle times but this cannot occur until thermal equilibrium at $(20 \pm 5)^{\circ} \mathrm{C}$ has been achieved

\section{Test System Descriptions}

\section{Zhejiang University Test System}

The test system used for this RR was an existing system at Zhejiang University's Institute of Process Equipment. The system was comprised of components and hardware in harmony with the requested system provided in the RR requirements. The following schematic (Figure 3) represents the specific arrangement of hardware.

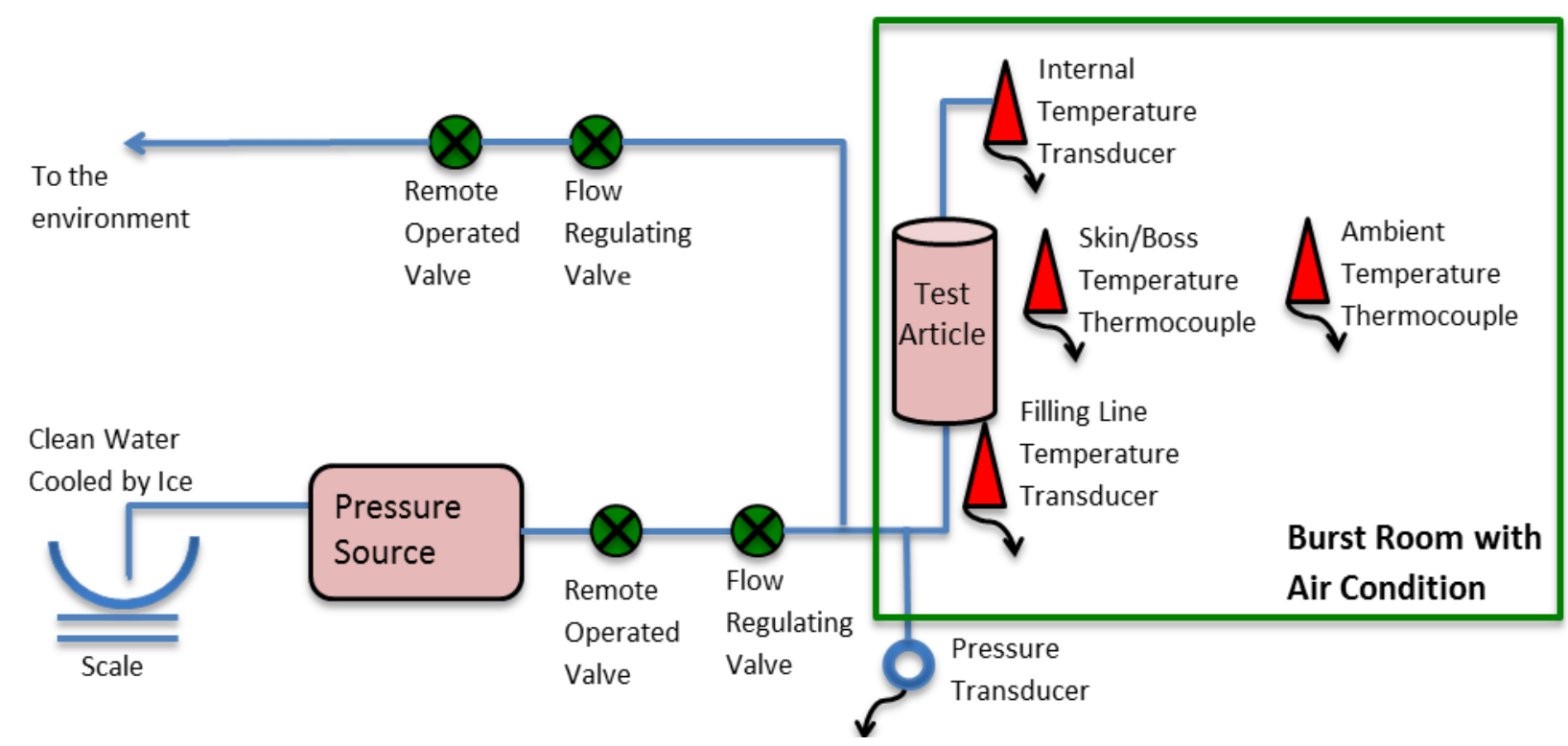

Figure 3: Zhejiang Test and Measurement System Block Diagram

Similar to the NASA-WSTF facility the Zhejiang University system was installed in a test bunker (Burst Room noted in Figure3) capable of withstanding a tank failure should one occur. The tests were operated from a remote location and under total computer control. No personnel were present in the test bunker when the vessel was pressurized. This was done for safety concerns as well as precise control over the experiment.The pressurization source was a multi-stage pump capable of producing pressures up to $90 \mathrm{MPa}$. The designed flow rate of the system is $60 \mathrm{~L} / \mathrm{min}$. Prior to beginning tests, the COPV was filled with water by turning the COPV on end filling the COPV and pressurizing the line by hand. Once the COPV was filled with water stagnation point end fitting was screwed into place. The COPV was then laid down horizontally and chocks were placed to prevent the vessel from rolling. During the test clean water was pumped into the COPV and when the target pressure was reached, the water pressure was relieved releasing the working fluid to the environment through a separate pipe located downstream of the pump and upstream of the COPV. The first tank test was performed during the winter and precooling of the pump inlet water was not needed.

However, the second tank test was performed in the summertime where temperatures would exceed $25^{\circ} \mathrm{C}$. During this test series, the water entering the pump was pre-cooled with ice. 
The COPV was placed in a test bunker approximately 20 meters downstream of the pump system. The ambient cell temperature was controlled via an air condition when cooling was needed and a resistive heater-style element with a fan when the test cell needed to be heated up.

Initially, the instrumentation for this test system consisted of a pressure transducer located downstream of the pump, and four thermocouples located on:

- The inlet end boss connection,

- The back end boss plug,

- The external skin,

- And the ambient test cell.

It is important to note that for the second tank testing the location of the inlet end boss thermocouple was changed as a result of a US-China face-to-face technical interchange meeting. This location change will be discussed later in this document.

Photographs of the instrumentation and other hardware are shown in Figures 4-6.

For the second tank test, two additional thermocouples were implemented,

- Working fluid in the fill line,

- And working fluid inside the tank.
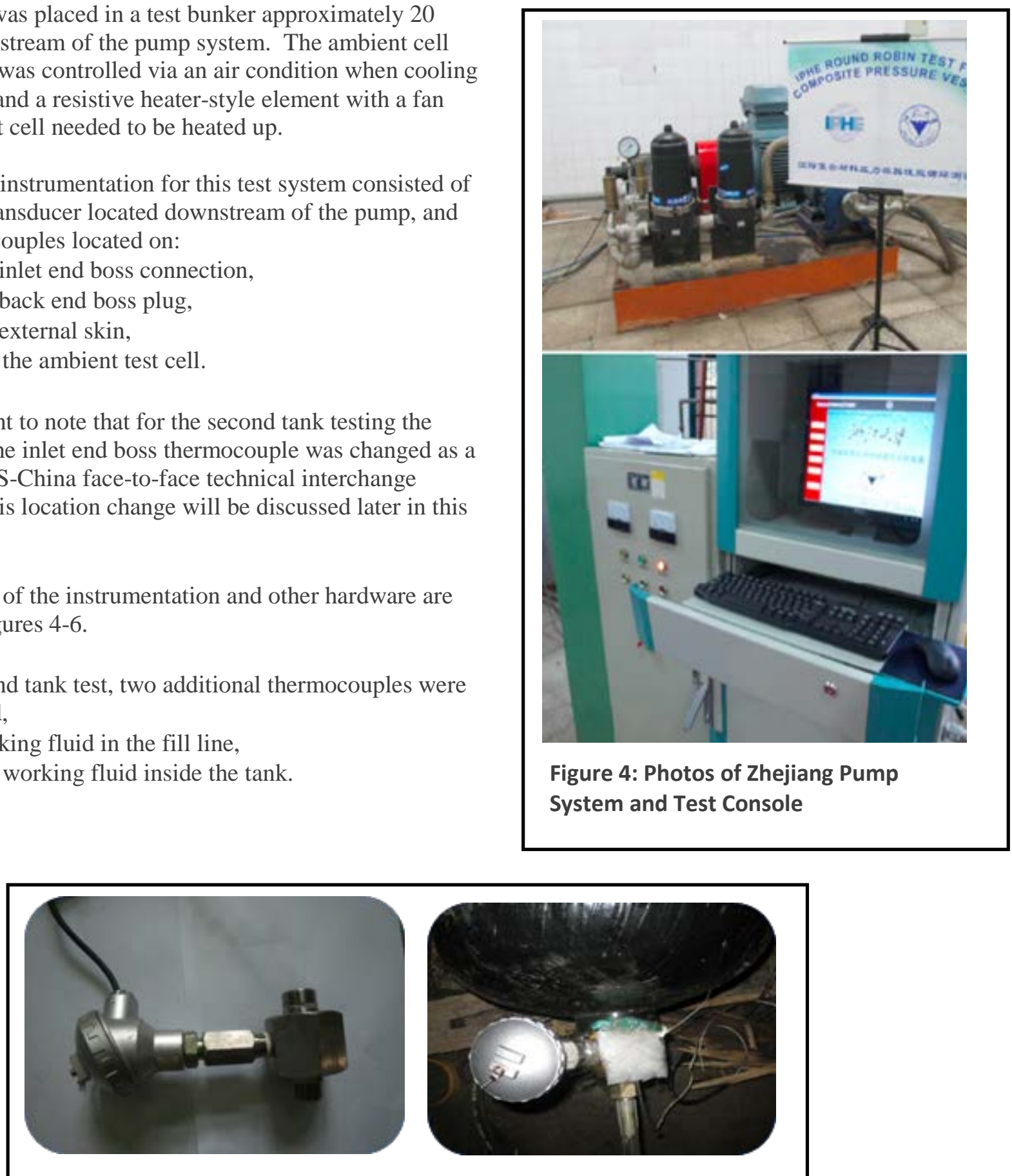

Figure 5: Temperature measurement of the water in the fill line 

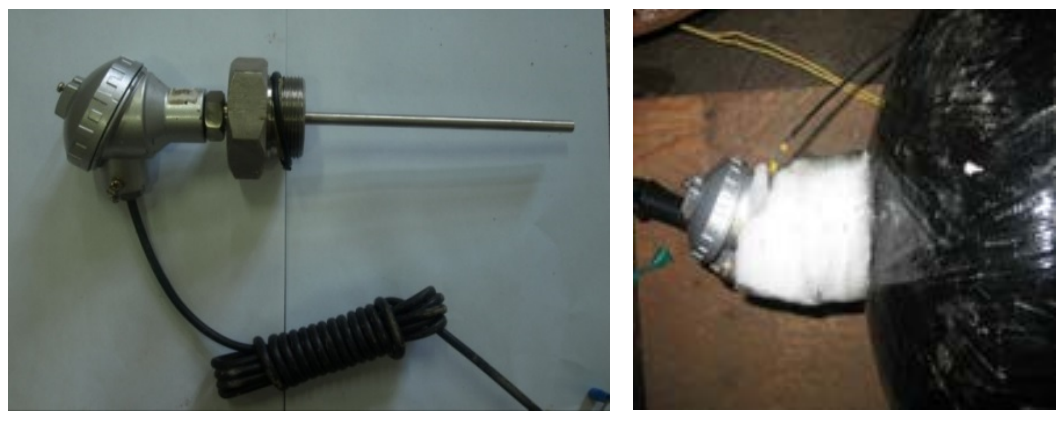

Figure 6: Temperature measurement of the water inside the tank

\section{NASA-WSTF Test System}

The test system used for this RR was assembled from used COPV hydraulic test pressurization equipment. A schematic of the NASA - WSTF system is shown in Figure 7. The NASA-WSTF system was also comprised of components and hardware in harmony with the requested system provided in the RR requirements. The system was installed in a test bunker capable of withstanding a tank failure should one occur. The tests were operated from a remote location and under total computer control. No personnel were present in the test bunker when the vessel was pressurized. This was done for safety concerns as well as precise control over the experiment.

Additional features in the pressurization and control system were implemented that were not specifically called out in the RR TMMP requirements. The test system was automated to track the temperature of the fluid inside of the test article and control the test system. This additional feature ensured that temperature limits were not

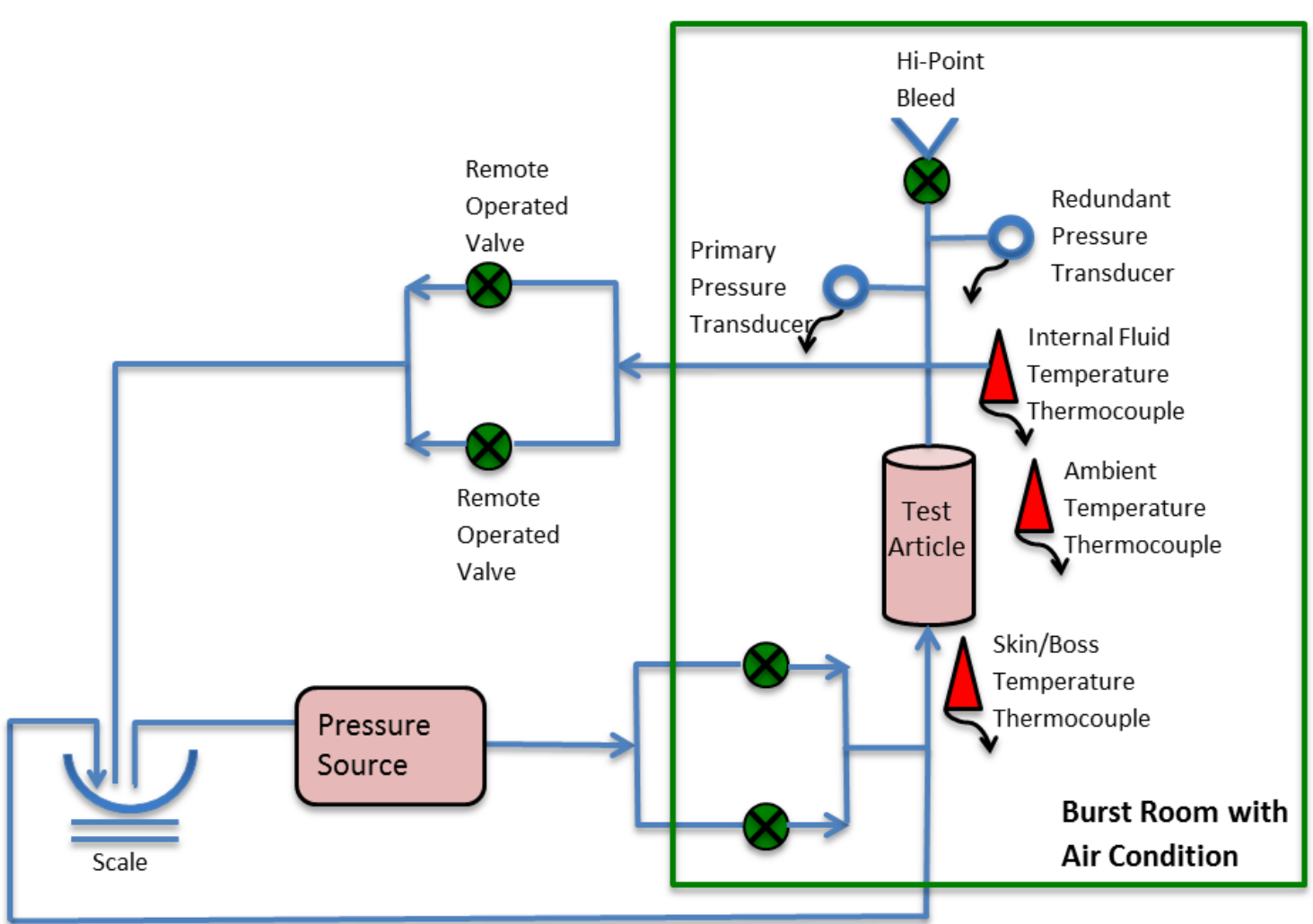

Figure 7: NASA-WSTF Test and Measurement System Block Diagram 
exceeded. The system was also automated with fluid control logic that would lock up the pressure in the tank if any pressure excursions or anomalies were encountered. The system was constructed as a closed-loop system capable of recirculating all the water used.

The pressure was delivered to the test article using a three-headed pump system capable of delivering a maximum outlet pressure of $68.9 \mathrm{MPa}$.

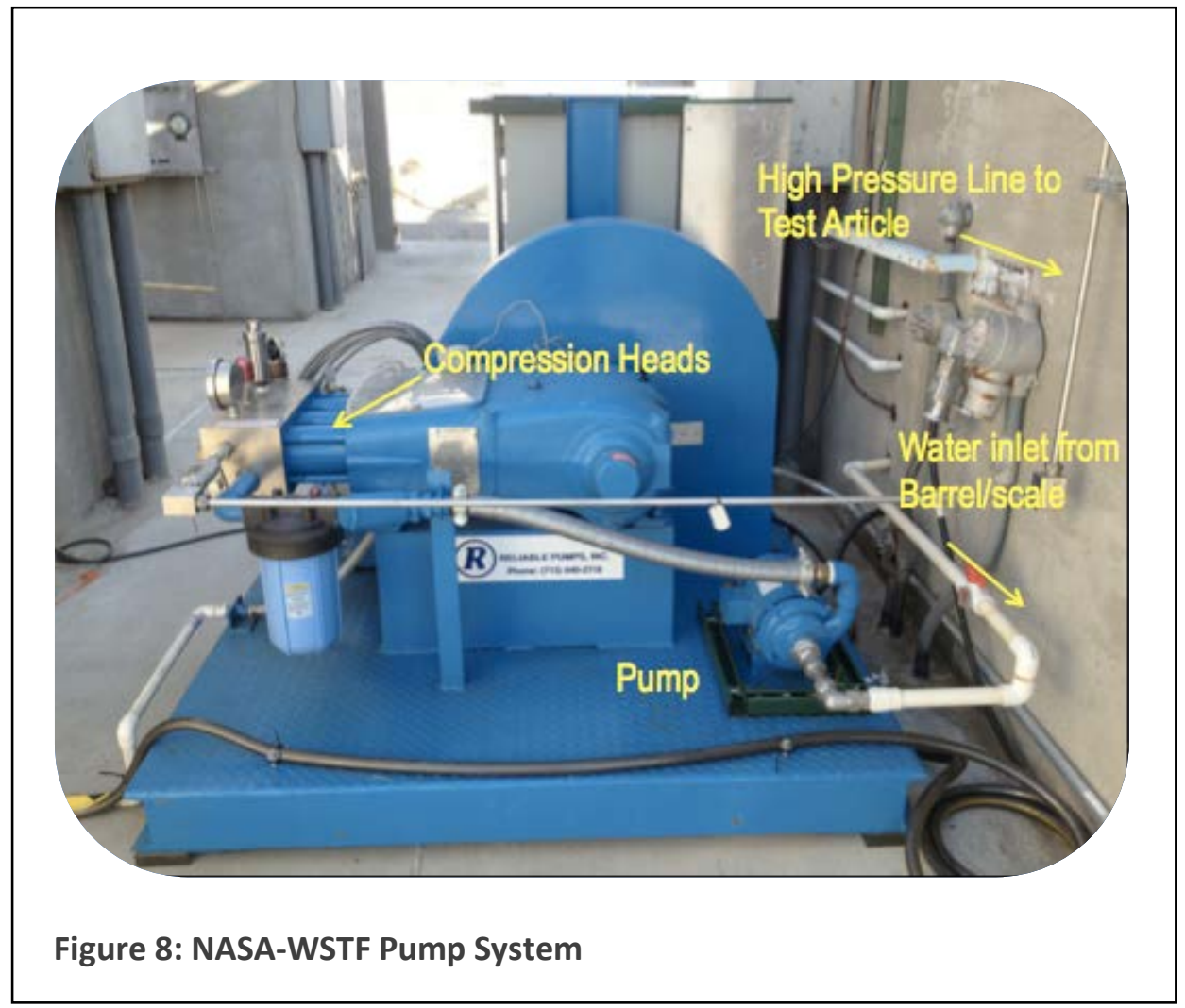

Fluid flow was controlled via electronically actuated backpressure regulators, (BPRs). Each of the "Remote Operated Valves" in the schematic in Figure 7 is a BPR. The BPRs are throttled opened and closed by the test computer to achieve a desired pressurization rate as read on the test article static pressure transducer. This system was configured with two independent BPRs upstream and downstream of the test article. The computer control logic was programmed to control pressurization, hold, and depressurization rates very accurately. This configuration was chosen for redundancy however became very convenient when this facility was asked to characterize fluid heating rates at various pressurization profiles.

The test article was placed approximately 10 meters downstream of the pump and roughly 5 meters downstream of the BPRs. The test article was installed in a vertical orientation with a clamping fixture holding the bottom COPV boss (Figure 9). The top boss was restrained to prevent tipping or falling of the bottle but was allowed to expand as the vessel was being pressurized. Fluid flow direction was controlled to flow in from the bottom boss fitting, through the COPV, and then out the pitot tubeend cap to be returned to the storage barrel.

Pressure measurements in the COPV were made through apitot tube plug/end cap. The end cap(Figure 10) contained four designed ports:

- $\quad$ Static pressure measurement port

- Dynamic pressure measurement port

- Fluid temperature port

- Flow-thru/return flow port. 

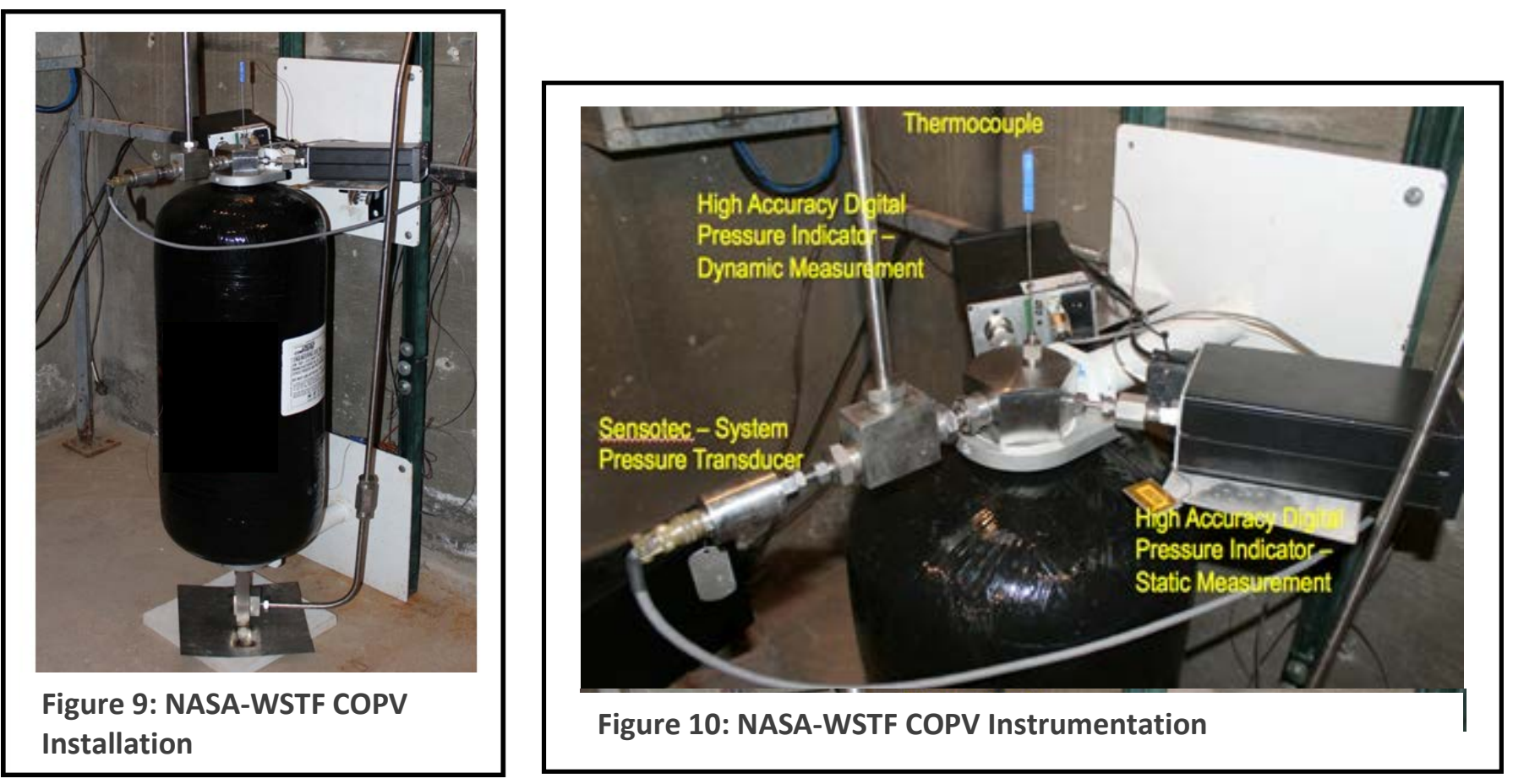

The pitot tube(figure 11) was constructed with a concentric tube design where the dynamic pressure port was positioned facing the fluid flow path. Figure 11(b) is a close-up of the tip of the pitot tube and the entrance to the dynamic pressure port. The static pressure port taps are drilled normal to the fluid flow direction and are seen best in Figure 11(c). The pitot tube/end cap was installed into the top end of COPV and the associated instrumentation is visible in Figure 10.

The water exiting the COPV was returned to the source water barrel (Figure 12)for recirculation in the system. The source barrel was placed on a scale to monitor the system for leaks.

A recirculating liquid chillerwas incorporated in the test system to aid in removing the heat introduced from the work being performed by the pump. The chiller's effects did allow from more cycles to be run consecutively before the upper temperature limit was met. The chiller was not able to cool the test media fast enough at the high cycle rate test cases; the upper temperature limit was reached resulting in pausing the testing.

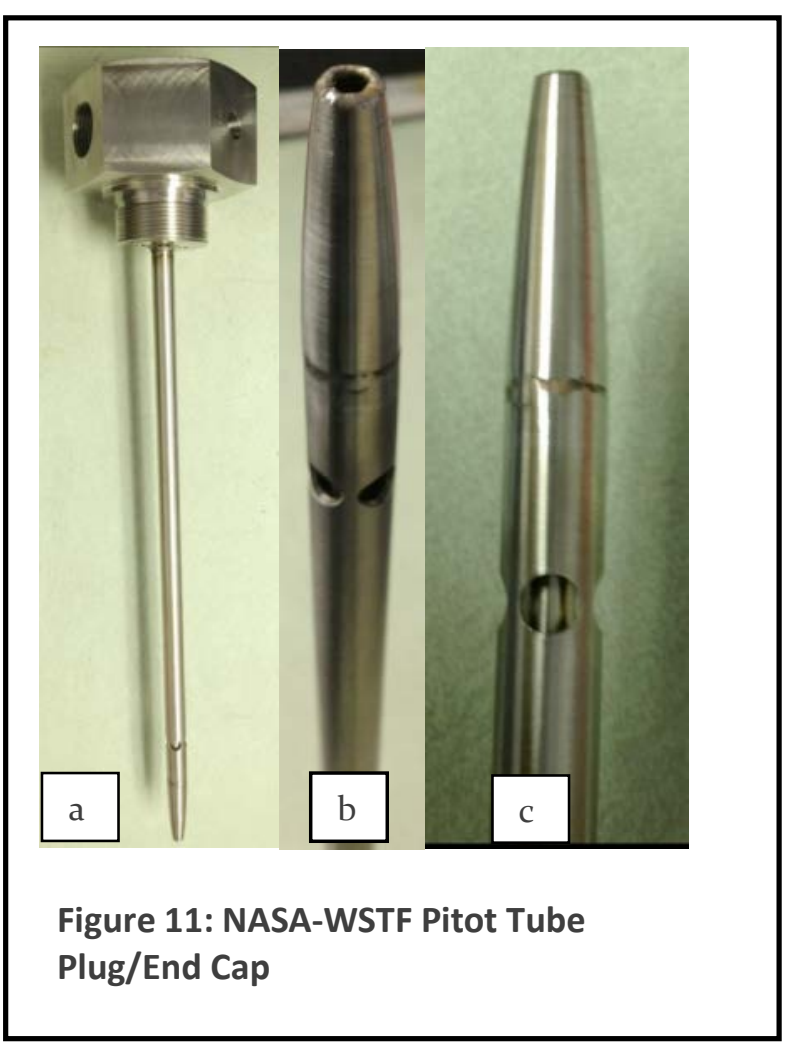




\section{Summary of Testing}

The TMMP required that 15,000 cycles be performed on each COPV. Both test facilities have completed the required cycling on one vessel and China has completed all cycles on the second vessel. Enough cycles were performed on each of 4 configurations to understand the temperature vs time behavior for the NASA-WSTF system.

The Zhejiang test system is capable of cycling the vessel at a rate of 6 cycles/min, which is the upper end of the TMMP requirement. See figure 13 for a typical pressure trace. For tank 1 cycling was halted after every 100 cycles to ensure that the temperature was maintained within the specification. During testing of the second COPV, pressure cycles continued until the temperature of the fluid inside the vessel reached the upper bound of $25^{\circ} \mathrm{C}$.

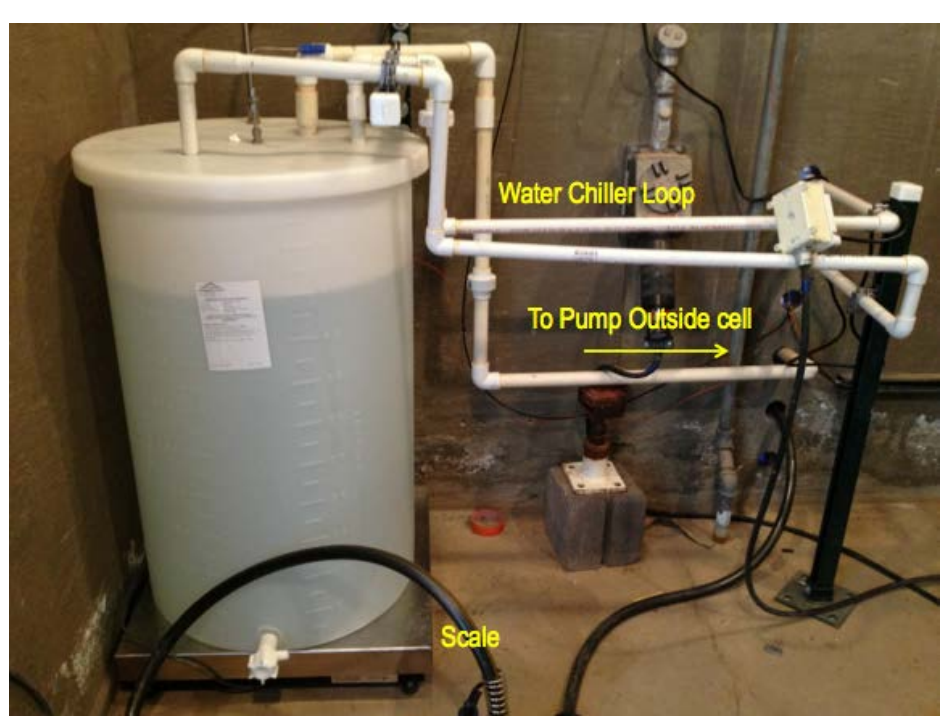

Figure 12: NASA-WSTF Water Storage / Return Instrumentation

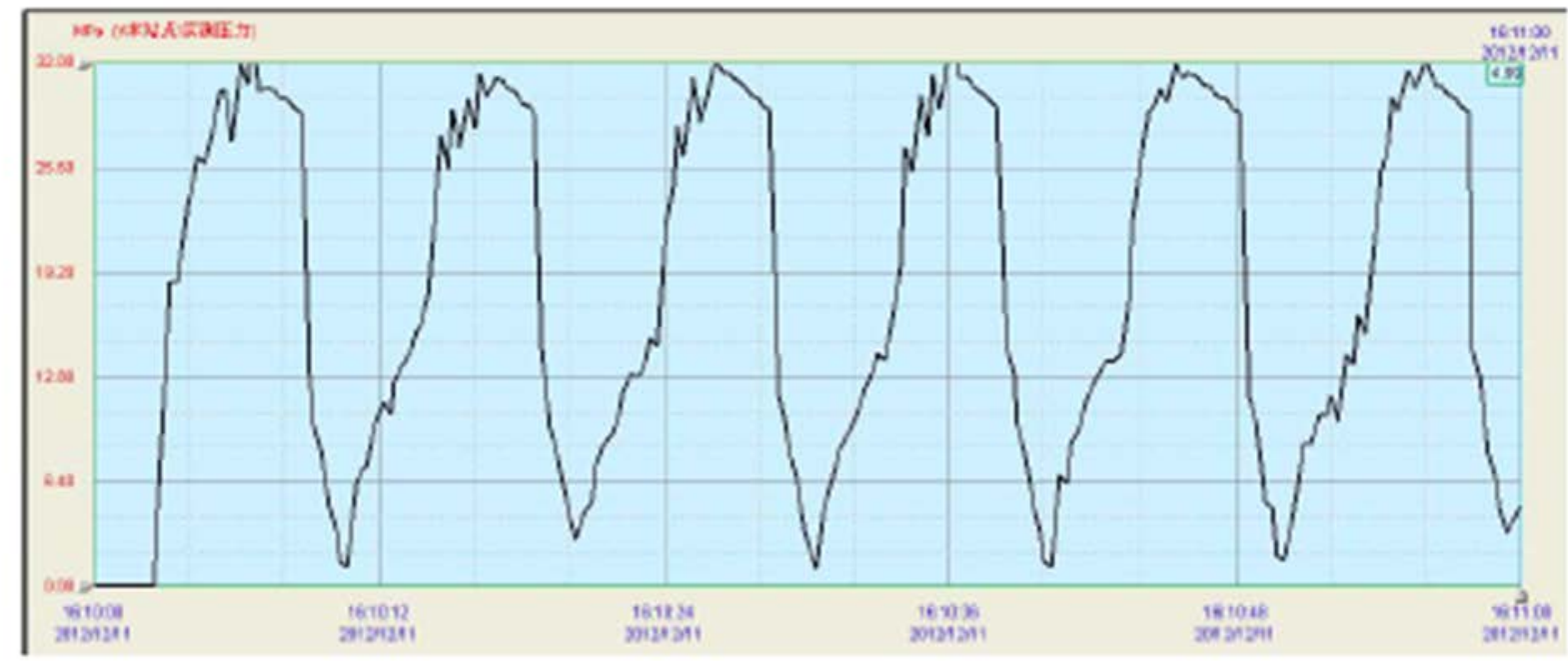

Figure 13: Typical Zhejiang University Pressure Profiles (6 cycles /min) 


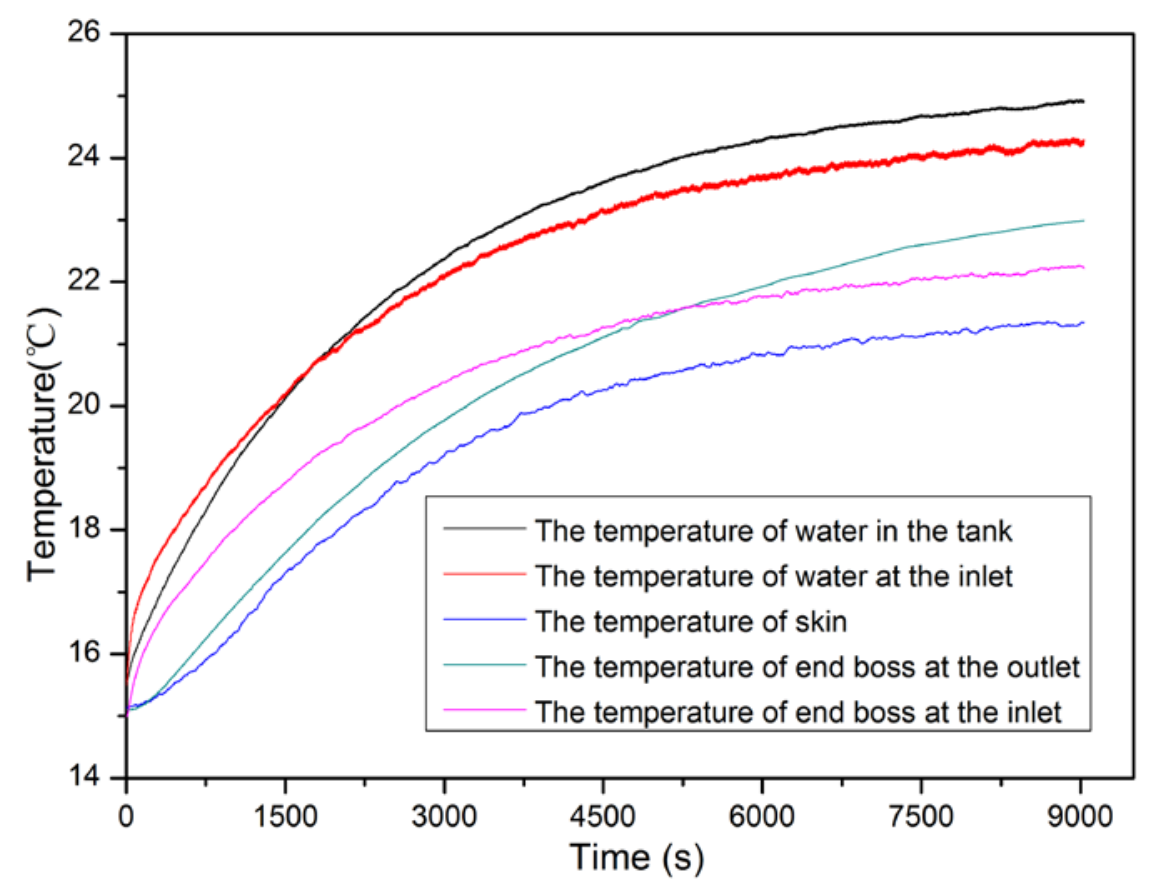

Figure 14: Typical Zhejiang University Temperature Profile for tank 2.

For testing of the second tank at the start of the test each day, the temperature of the ice cooled water would begin at the lowest condition stated in the TMMP requirements, $15^{\circ} \mathrm{C}$. After roughly 910 cycles, the temperature would reach the upper specification $25^{\circ} \mathrm{C}$ and the testing would be halted. Figure 14 shows a typical temperature time profile taken with the second tank. Only 800-1000 cycles were performed per day to control the overall temperature rise in the system and to reset the temperature to the lower specification for the following day.

After the cycle testing both tanks were pressurized until failure occurred. One tank failed due to an O-ring failure on the boss fitting connection and the other failed due to a burst event. Tank 1 leaked at a pressure of 76.2 MPa, while Tank 2 burst at a pressure of $78.7 \mathrm{MPa}$ for a factor of 3.07 and 3.17 above NWP for tank 1 and tank 2 respectively.

The design of the NASA-WSTF test system facilitated exploration of various pressurization rates and theireffect on introducing heat into the test media. A typical pressure trace for the NASA-WSTF is shown in Figure 15. Two different pressurization rates with two different flow conditions were tested:

- One cycle per minute in an open system (flow through) configuration

- One cycle per minute in a closed system (dead-ended) configuration

- Three cycles per minute in an open system (flow through) configuration

- Three cycles per minute in a closed system (dead-ended) configuration 


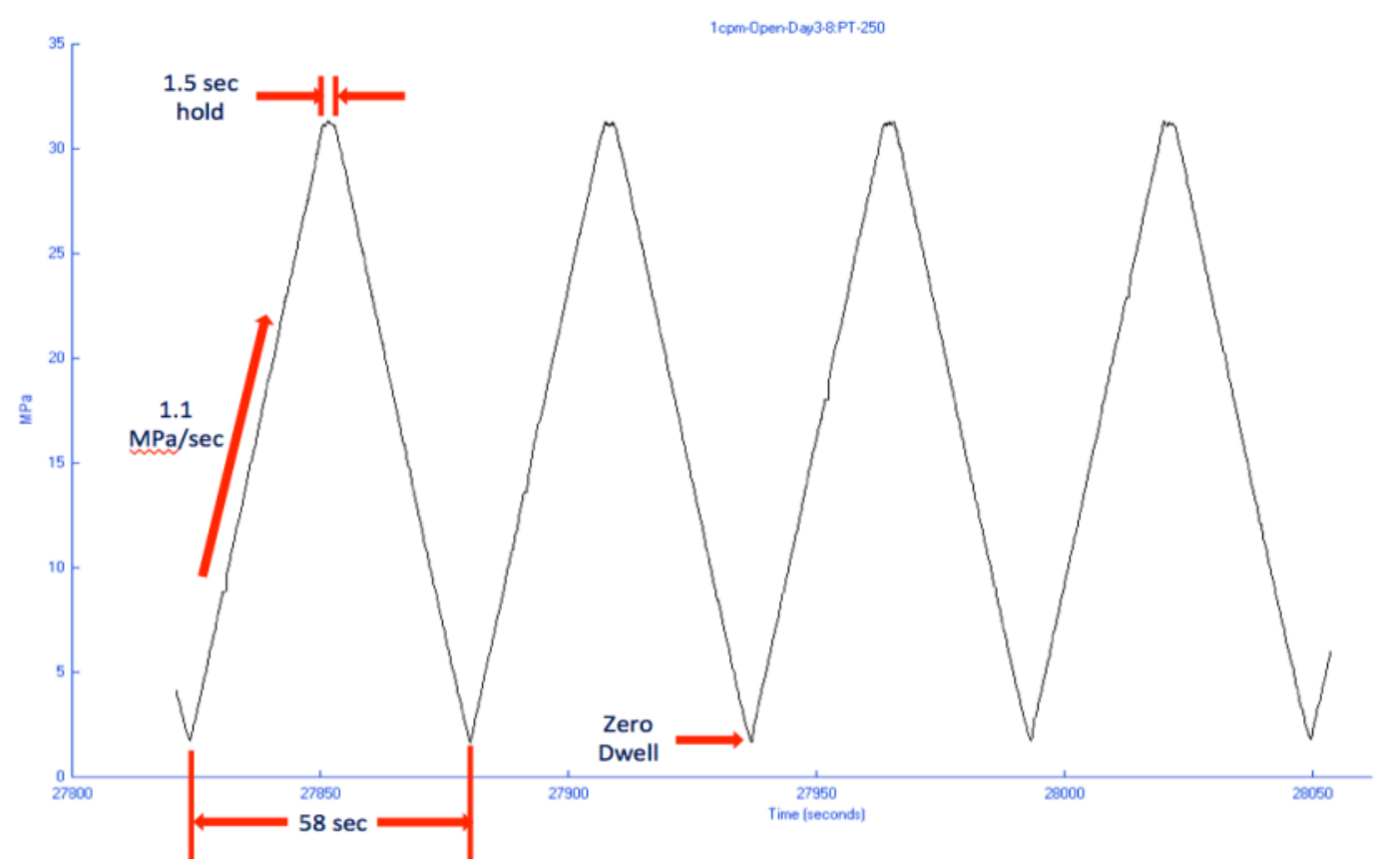

Figure 15: Typical NASA-WSTF Pressure Profile (1 cycle / min)

For the open system test configuration the BPRs upstream of the COPVwere kept in the open position such that the test media was allowed to flow through the test article and the test system. The desired pressurization rate was maintained in the test article and throughout the system by the pressure pump and a downstream BPR.

For the closed system test configuration the BPRs downstream of the COPV were closed and the upstream BPR was throttled to allow the system pressure to bleed down at a controlled rate. In addition, the test media is only allowed to flow through a small portion of the test system. The desired pressurization rate is maintained in the test article and throughout the system by the pressure pump and a designated back pressure regulator.

Comparison of the pitot tube data shows that the pressure difference between static and dynamic pressure in a flow through condition is minor. The plot in Figure 16 shows the dynamic pressure as the red trace and the static pressure as the black trace. The difference between both curves is at most $0.26 \mathrm{MPa}$. Note: under these conditions the test medium (water) is not incompressible; the tank volume will increase with increasing pressure. Note that in this configuration the pitot tube was mounted in the end boss in the downstream location near the exit of the tank. In the flow through configurationthe test medium is always flowing through the system. Due to the expansion of the tank under pressure and compressibility of the test medium the flow rate through the system changes. The flow rate (at the exit where the pitot tube is located) during pressurization is lower than that at the peak pressure hold which is lower than that during depressurization $\left(\dot{\mathrm{M}}_{\text {Pressurization }}<\dot{\mathrm{M}}_{\text {Pressure hold }}<\dot{\mathrm{M}}_{\text {depressurization }}\right)$ As the pressure increases the test medium compresses and the tank volume increases and hence, the flow rate at the exit decreases. At the peak pressure hold the flow rate is constant at the peak of the pressurization portion of the cycle, resulting in an increase in the difference between the dynamic and static pressure measurements as compared to the pressurization part of the cycle. During the depressurization part of the cycle the test medium is expanding and the tank volume decreasing the flow rate continues to increase again resulting in an increase in the difference between the dynamic and static pressure. On a relative scale the difference between the dynamic and static pressure is minor (0.26 MPa out of $25 \mathrm{MPa}$ or about $1.04 \%)$. It should be noted that on an absolute scale this represents about 2.6 atmospheres. Care needs to be taken to ensure that the static pressure is measured without any dynamic pressure effects. 
A plot of the static versus dynamic pressures for the closed system configuration is not shown because both curves fell on top of each other.

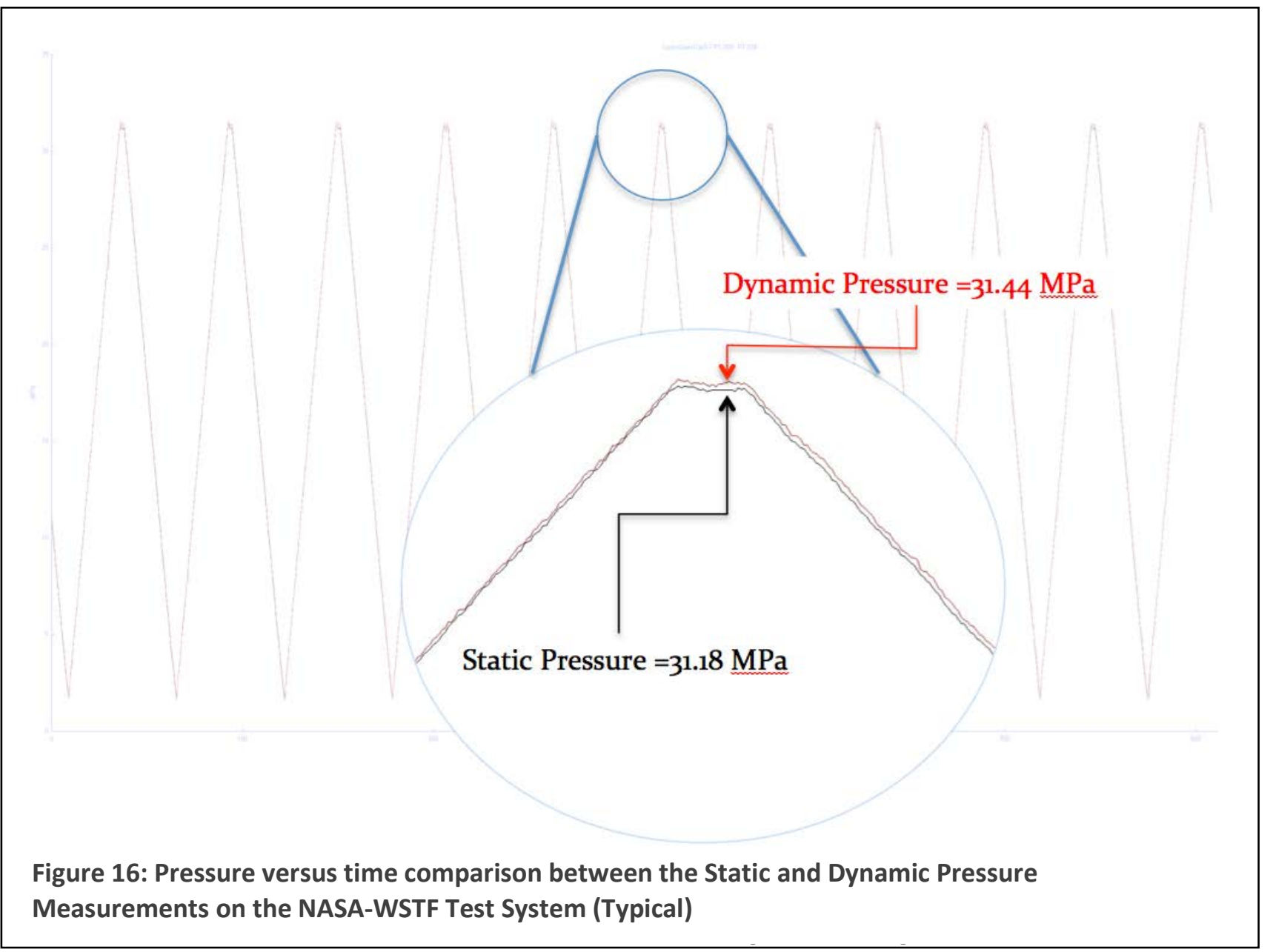

Lessons Learned/Major Findings

Both the Zhejiang University and NASA-WSTF test systems experienced test media heating as a result of the pressure cycles.Contrasting the similarities and differences between the two systems provides insight into design considerations of future hydraulic pressurization systems. The Zhejiang University test system was designed as a single-pass system with a dead-ended line connecting to the COPV. This method minimized heating of the fluid in the dead-ended line as cold ice water was fed to the pump and then released to the environment as the system pressure was reduced.

One of the configurations for the NASA-WSTF test system was a closed-loop recirculation system with a chiller loop cooling the water in the supply/return barrel. The system's redundancy allowed for both dead-ended and flow-through conditions to be evaluated. This closed-loop system produced a significant amount of fluid heating that had to be removed in order to stay within the test parameters.

The recirculating liquid chiller was optimized for 1 cycle/min flow-through conditions. For this condition, the heat exchanger was able to remove the heat such that the temperature asymptotically reached a steady valued enabling a continuous operation (see Figure17). Operating the system at the higher cycle rate of 3 cycle/minute was out of design for the Chiller. Hence the chiller was not able to cool the water fast enough and the upper temperature limit was reached resulting in a test system shutdown. A lesson learned that should be applied to any 
future test system is that proper sizing of a heat exchanger is critical for development of future closed-loop and open-loop systems to enable continuous operation.

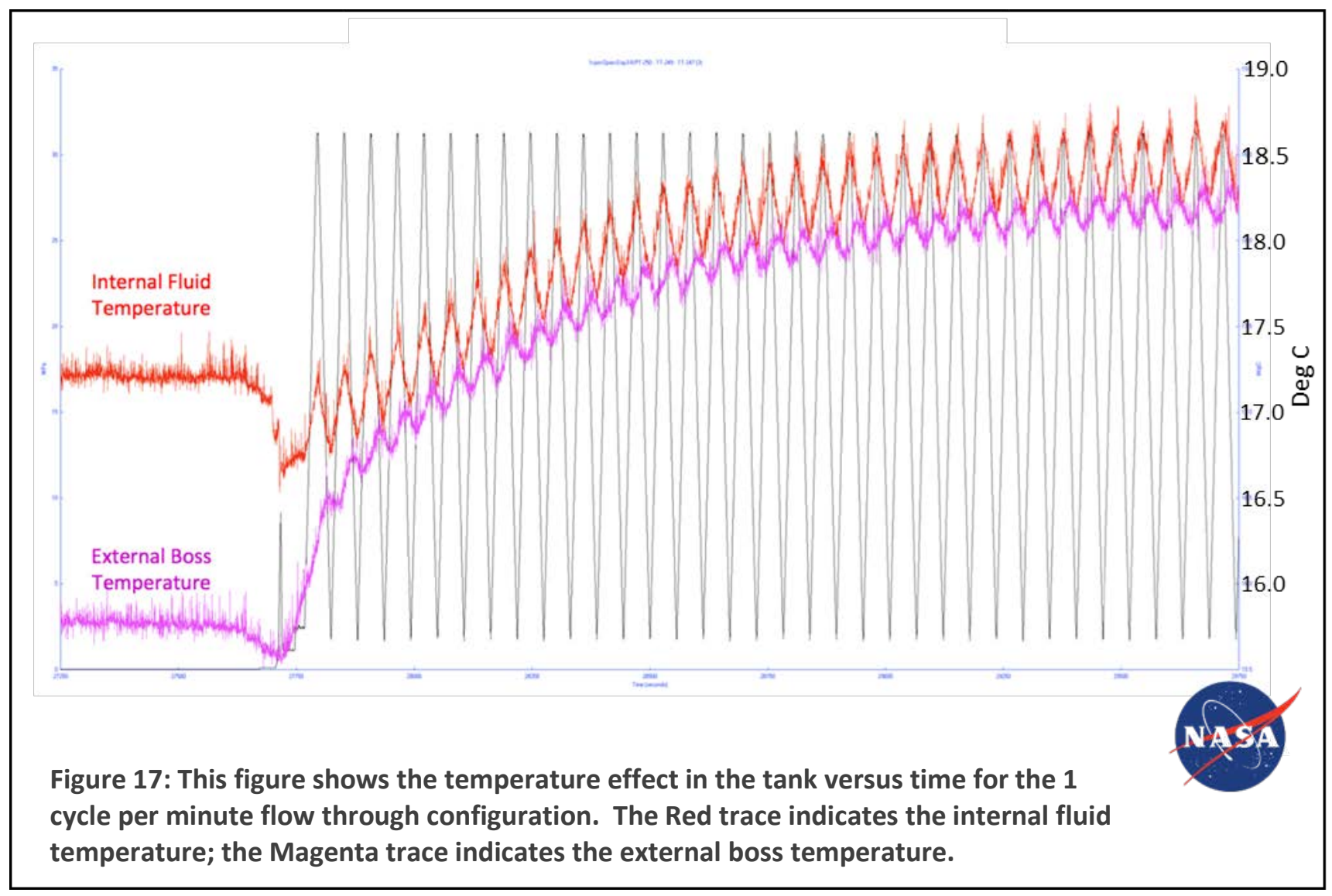

Another significant lesson learned deals with proper location of the thermocouple measurements. Comparisons of the charts shown in Figure 18from the NASA-WSTF configurations indicate that temperature measurement taken at the test article boss and the measurement taken from the fluid inside of the test article can differ from each other in a range of 0.5 to $1^{\circ} \mathrm{C}$. The red traces are temperature measurements of the internal fluid. The green traces are external boss temperature measurements. The data in figure 18 show that the temperature of the internal fluid rises at a greater rate than the temperature measured at the boss in all configurations except the FlowThrough 1 cycle/min where the temperature was well controlled by the chiller; however even for this case the boss temperature lagged the temperature of the internal fluid. 

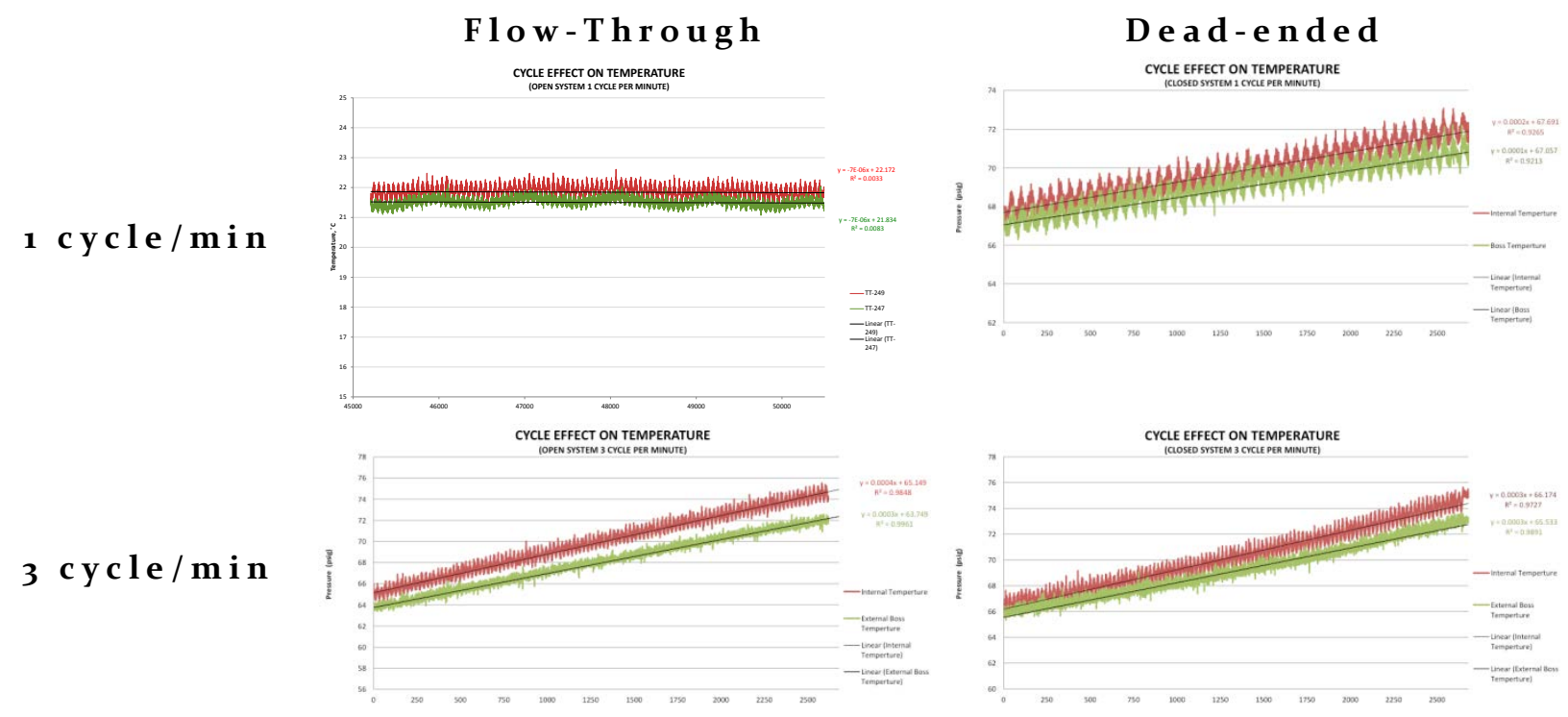

Figure 18: Comparison of NASA-WST Fluid and Boss Temperature for the First Tank. The Red trace represents the internal tank fluid temperature, the Green trace represent the external boss temperature

During the face-to-face meeting at Zhejiang University the placement of the boss thermocouple was discussed. The data in Figure 18 shows that solely measuring the boss temperature could result in inaccurate estimates of the actual fluid temperature in the COPV. Implementation of internal fluid temperature thermocouples and re-position of the inlet boss thermocouple was imperative to ensure proper measurement of the fluid conditions. Both facilities agreed to place the boss thermocouple in direct contact with the metal boss as close to the dome region of the COPV - but without touching the composite dome, use heat transfer grease to improve the thermal contact between the boss and the thermal couple and shield/insulate it from the external environment.

Also, during cycling of the first test article, it was discovered that the ambient condition surrounding the test article influenced the temperature measurement taken at the boss. Adhering to the TMMP a thermocouple was positioned on the inlet boss of the test article. The thermocouple was placed onto a clean area of the boss and held into place with adhesive. The backside of the thermocouple was left exposed to the ambient conditions. While reviewing the data from the first test at NASA-WSTF and Zhejiang University, a temperature increase was seen on the boss thermocouple when the test cell's HVAC would turn on to warm the cell. The temperature spike caused by the HVAC system did not appear to affect the thermocouple used to measure the internal fluid temperature for the NASA-WSTF. Figures 19 and 20show the effect the HVAC had on the boss thermocouple. The black line is the test cell ambient temperature. 


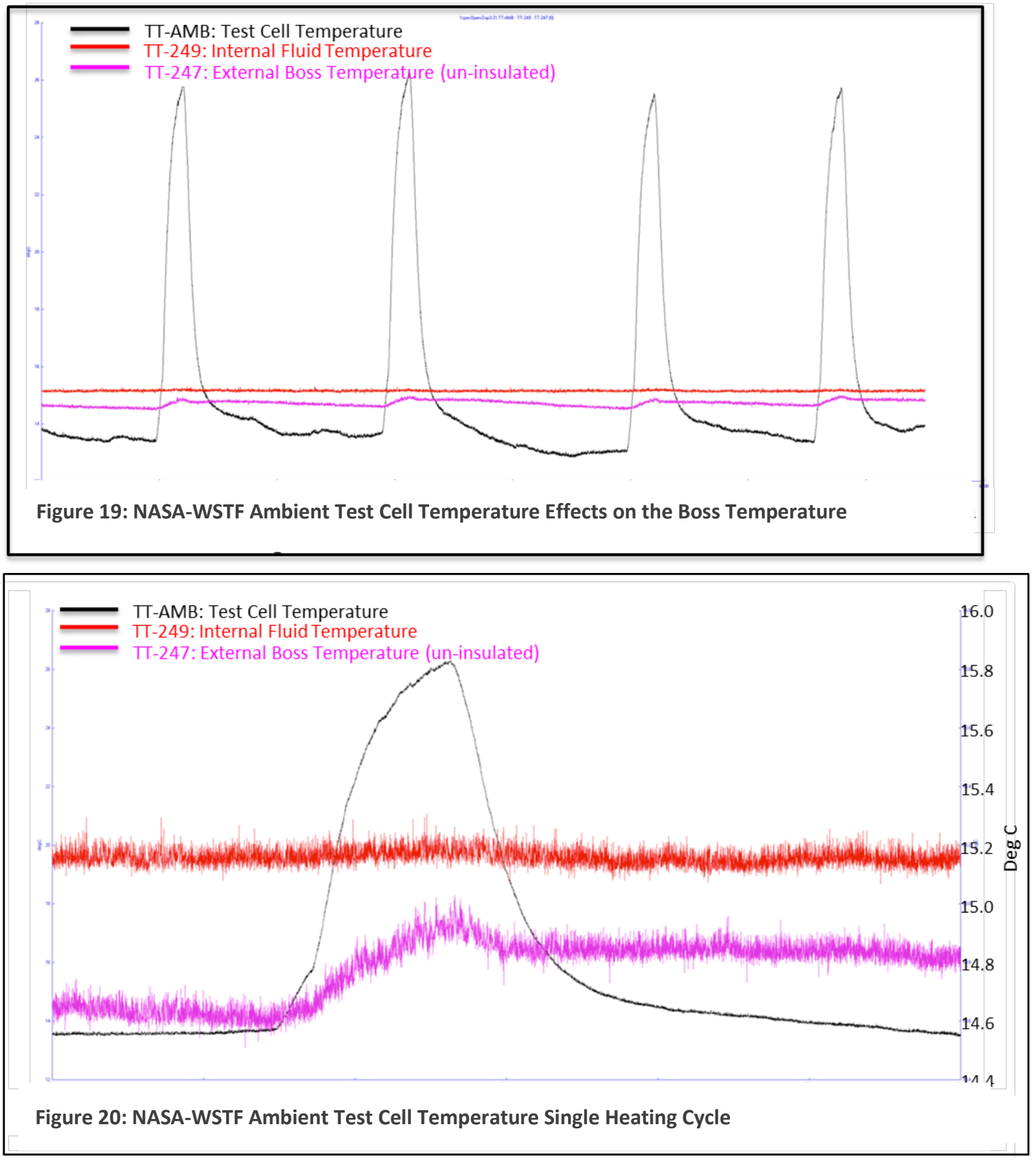


The data sets for NASA-WSTF tank 2 for 1 cycle/min and 3 cycle/min were complete enough that a comparison between the temperature evolution and cycle rate can be made. Shown in table 1 are the results of a linear regression from the temperature data from these two runs.

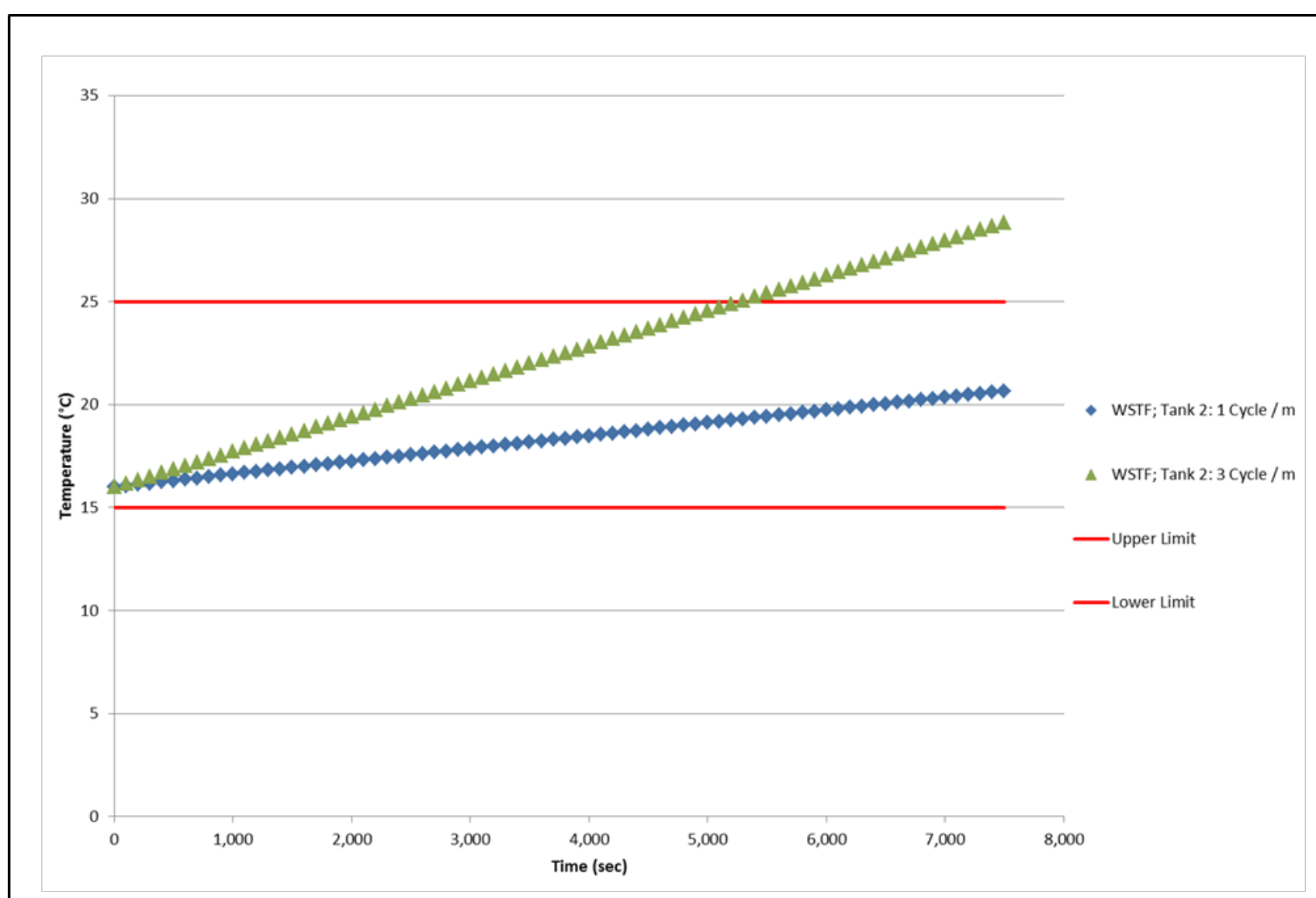

Figure 21: Comparison of temperature versus time for the NASA-WSTF configuration.

\begin{tabular}{|l|c|}
\hline & $\begin{array}{c}\text { Slope of Linear } \\
\text { Regression } \\
\left({ }^{\circ} \text { C/sec }\right)\end{array}$ \\
\hline Condition & Tank 2 \\
\hline 1 cycle/min (dead-ended) & 0.00062 \\
\hline 3 cycle/min (dead-ended) & 0.00711 \\
\hline
\end{tabular}

Table 1: Comparison of the Linear Regression Results for each flow condition. 


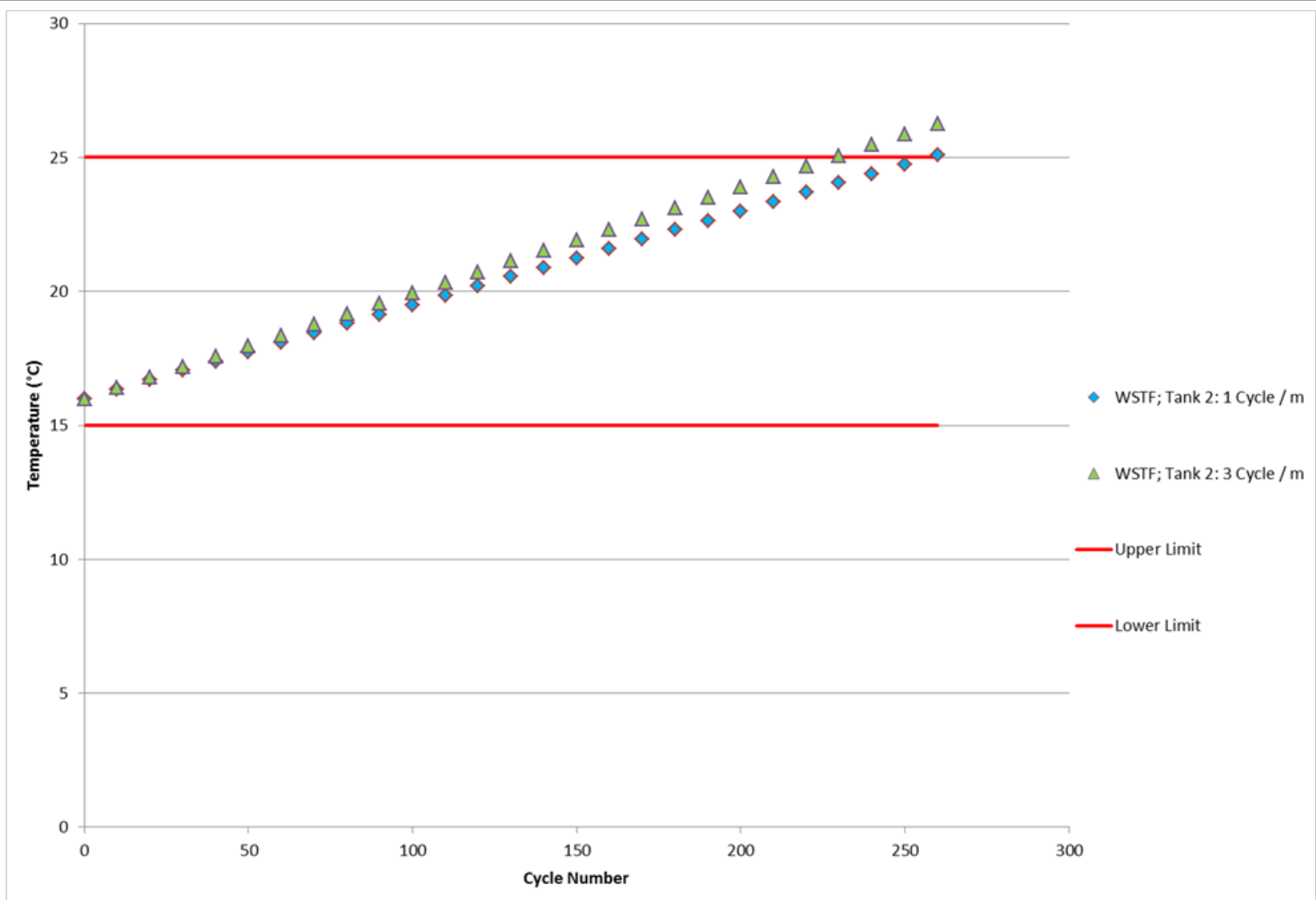

Figure 22: Comparison of temperature versus cycle number for the NASA-WSTF configuration.

Using the values in the Table 1, plots were created to visually compare the predicted heating slopes for these two cycle rates

Figure 21 plots the average temperature increase with time, Figure 22 plots the same data but versus cycle number. Note that the slopes collapse to a single line for the tank 2, 1 and 3 cycle/minute runs. The 1 cycle per minute remains a little lower than that for the 3 cycle per minute probably due to heat transfer effects. Also, note that all the NASA-WSTF data reach the upper limit after about 250 cycles. This indicates that the upper limit on temperature will be reached at about 250 cycles regardless of the cycle rate. Assuming the tank is isolated from the pumps and other heat adding devices, the temperature rise in the tank should be a result of the net work performed on the working fluid and the tank, which is a function of the cycle not the repetition rate. At slower rates the effects of heat transfer to the environment will be more important - hence the lower slope for the 1 cycle/minute vs 3 cycle/minute.

The Zhejiang University data does not hit the upper temperature bound until cycle number 910. Recall, the Zhejiang University experimental configuration drew the input water from an ice cooled water bath. The test room was very well insulated from the environment, and hence it stayed at a constant temperature during the test.This setup yielded better thermal control over temperature of the input fluid for the dead-ended configuration than did the heat exchanger approach used in the NASA-WSTF system. To shield the boss temperature measurement from outside influences, during the second test, the backside of the boss thermocouple was covered with a piece of insulation. By ensuring that the boss thermocouple measurements were not compromised, a comparison of internal fluid and boss temperatures could be made.The insulation of the boss thermocouple prevented erroneous temperature measurements. 


\section{Summary, Conclusions \& Recommendations}

To address the lack of uniformity in tank qualification testing around the globe the IPHE RCSWG engaged in a Round Robin activity to establish a uniform test measurement method. Applying this uniform test method will help to ensure consistent results regardless of the test facility. The Round Robin was divided into two phases, Phase I, is a hydraulic cycle testing activity and Phase II is a pneumatic cycle testing activity. This manuscript reports on activities focused on Phase I. A Test Measurement Method Protocol (TMMP) was designed and executed at two different locations China, and the United States. As a result of this effort, we now understand the critical parameters to be measured and the method which these parameters need to be measured in order to ensure, consistency in complying with any specified qualifying test. This work also highlighted gaps in understanding type IV tank behavior as a function of various parameters needed to be controlled to ensure a correctly executed test.

The results of the IPHE RCSWG TMMP round robin have demonstrated that the requirements outlined in the TMMP that wereextracted from SAE J2579, EIHP Rev 12B, and the GTR Phase 1, yielded similar test results from two separate test locations. Both the Zhejiang University and NASA-WSTF locations were able to successfully perform 15,000 cycles on Type IV COPVs and obtaincomparable test data.

The results of the TMMP provided insight into what factors influence the internal fluid temperature of the test article and perhaps most importantly has shown which temperature measurements were critical in instrumenting to successfully perform this test. It is apparent that the higher the cycle rate the faster the temperature rise of the working fluid compared to a slower cycle rate, however, temperature rise on a per cycle basis appears to be roughly a constant. This last conclusion is system dependent. It was observed that test media temperature can be influenced by controlling both the ambient temperature and the temperature of the test media entering the pumping system. It is also noted that the performance of the test is dependent on the test configuration and test parameters. While understanding the temperature rise as a function of cycle number is valuable in controlling and calculating the rise over a given number of cycles, this analysis should not be used to extrapolate between systems and is only valuable for a single system configuration and operation.

It was not the intent of this round robin to develop a tank qualification sequence or to qualify laboratories to execute test sequences. The test measurement protocol pressurization and temperature requirements were based on requirements from the above consensus standards as well as from some manufacture's recommendations for avoiding certain pressure/temperature regimes. The protocol was in no way intended to simulate real-world automotive conditions. The vessels tested did not fail prematurely and perhaps additional study could be performed to understand the critical parameters that affect the fatigue life of Type IV vessels particularly the behavior of the liner.

By far and away the most challenging aspect of this work was the control of the temperature.It is not clear how the temperature increase affects the fatigue performance of the tank. The physics describing type IV liner failure when the tank is at elevated temperature and atmospheric pressure are not at all understood. This is a critical issue to understand, and needed to ensure that tank qualifying sequence testing is executed in such a manner that one does not artificially impose conditions not seen in the field and hence test the tank under unrealistic conditions.

Recommendations for future work:

- Studythe lower limit pressure and fluid temperature inside the tank and their effects on fatigue life and the liner behavior,

- $\quad$ Conduct further modeling and testing of the mechanisms influencing the temperature variations during cycle testing.

- Investigate the effect of temperature variation on tank fatigue performance (particularly the liner) needs to be investigated 
To fully understand the effects of various test parameters influencing the temperature variation and the response of the tank system to this variation additional testing and modeling is necessary.

\section{Acknowledgements:}

We appreciate the financial assistance of the U.S. National Aeronautics and Space Administration (NASA); the U.S. Department of Energy, Fuel Cell Technology Office, Safety Codes and Standards Program; the Ministry of Science and Technology, Peoples Republic of China; and Universidade Estadual de Campinas/Brazilian Reference Center for Hydrogen Energy, Brazil. We thank Norm Newhouse from Hexagon Lincoln for supplying the tanks used in this investigation. We thank Aaron Harris,previously at Sandia National Laboratories U.S., for his assistance.

\section{References}

1. European Integrated Hydrogen Project (EIHP) CGH2R rev 12b (EIHP rev 12b) - (GRPE Informal Group: Hydrogen/Fuel Cell VehiclesDraft ECE Compressed Gaseous Hydrogen Regulation Revision 12b, 12.10.03)

2. SAE J2579 rev 2 draft,

3. Global Technical Regulation (GTR) for Hydrogen Fueled Vehicles (ECE-TRANS-WP29-GRSP-201212e) 